\title{
The Investigation of Treatment Effects on Serum Biochemical Parameters in Bladder Cancer Diseases
}

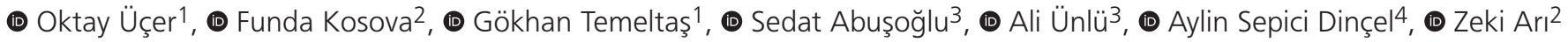 \\ ${ }^{1}$ Celal Bayar University Faculty of Medicine, Department of Urology, Manisa, Turkey \\ ${ }^{2}$ Celal Bayar University Faculty of Medicine, Department of Medical Biochemistry, Manisa, Turkey \\ ${ }^{3}$ Selçuk University Faculty of Medicine, Department of Medical Biochemistry, Konya, Turkey \\ ${ }^{4}$ Gazi University Faculty of Medicine, Department of Medical Biochemistry, Ankara, Turkey
}

\begin{abstract}
Objective: The most common cancer in smokers is bladder cancer (BC). Intravesical chemo immunotherapies are used to lower the risk of progression in patients who are at risk. Bacillus Calmette-Guérin immunotherapy is the most effective adjuvant therapy discovered. We aimed to evaluate the levels of arginine, citrulline, ornithine, symmetrically dimethylated arginine (SDMA), N-monomethyl-Larginine. (L-NMMA) or asymmetrically dimethylated arginine (ADMA) in patients with BC, as well as their relationship with methylarginine.

Materials and Methods: Blood samples were collected from all patients $(n=30)$ and controls (group 1) prior to transurethral resection of bladder tumour (TURBT) (group 2), 20 days after TURBT (group 3) and at the end of intravesical immunotherapy 74 (group 4). The levels of serum methylated arginine were measured using ABSCIEX API 3200 tandem mass spectrometry system in positive ESI mode.

Results: In comparison to group 2, group 1's ADMA and arginine/total methylated arginine levels were 98 significantly lower ( $p=0.035$ and $p=0.049$, respectively), while SDMA/ADMA, L-NMMA and arginine/ADMA levels ( $p=0.001, p=0.008$ and $p=0.017$ ) increased, and no statistical difference was found for other parameters $(p>0.05)$. When compared to group 3, ADMA, arginine, citrulline, methylated arginines and L-NMMA levels in group 2 ( $p=0.035, p=0.001, p=0.015, p=0.032$, $p=0.032)$ increased, while SDMA/ADMA levels $(p=0.041)$ decreased.

Conclusion: The decrease in arginine and ADMA levels in non-muscle invasive BC patients is thought to be promising, and these markers may be useful in monitoring the diagnosis and treatment of patients.
\end{abstract}

Keywords: Bladder cancer, ADMA, arginine, citrulline, SDMA, L-NMMA

\section{Introduction}

Bladder cancer $(B C)$ is one of the most common cancers worldwide (1). The diagnosis of $B C$, the most common urinary tract tumour, is based on urinary cytology and white-light cystoscopy in patients suspected of having a bladder mass and haematuria (2). As a result, despite the fact that many drugs for $\mathrm{BC}$ have been developed, we need new agents for therapeutic and diagnostic purposes due to the toxicity and resistance caused by these drugs. Protein arginine methyltransferases (PRMTs) are enzymes that play a key role in important cellular events such as signal transduction and transcriptional activation and inhibition, by catalysing methylene residues $(3,4)$ and by transferring methyl groups from S-adenosyl-1-methionine to terminal guanidino nitrogen atoms (5). Arginine methylation can generate asymmetric NG, NG-dimethylarginine as well as type I (PRMT5 and PRMT7) symmetrical NG and NkenG-, while S-adenosylmethionine (PRMT 1, 2, 3, 4, 6 and 8), used as a methyl donor, is a posttranslational modification catalysed by PRMTs (6), which are the functions of dimethylarginine. Through signal transduction, transcription and mRNA splicing, arginine methylation facilitates protein-protein interactions and protein localisation $(7,8)$. There are three types of methylated arginine: monomethylated arginine (MMA), asymmetrically dimethylated arginine (ADMA) and symmetrically dimethylated arginine (SDMA) (9).

In general, histone ADMA is associated with active transcription, whereas histone SDMA is correlated with transcriptional repression (10). While histones are true PRMT substrates, the majority of nuclear arginine methylation is found in heterogeneous nuclear ribonucleoproteins, implying that PRMTs can also regulate gene expression post-transcriptionally (11).

Cite this article as: Üçer O, Kosova F, Temeltaş G, Abuşoğlu S, Ünlü A, Sepici Dinçel A, Arı Z. The Investigation of Treatment Effects on Serum Biochemical Parameters in Bladder Cancer Diseases. Bull Urooncol 2021;20(2):107-110 
After being converted to L-citrulline and dimethylarginine by hydrolysis (12), ADMA is obtained by arginine methylation in intracellular proteins by $\mathrm{N}$-methyltransferases (13) of Type I protein arginine.

As a result, arginine methylation and PRMT structural defects are linked to carcinogenesis, metastasis and drug resistance $(14,15)$. In light of this information, we believe PRMTs hold promise for cancer diagnosis and treatment. We aimed to evaluate the levels of arginine, citrulline, ornithine, SDMA, N-monomethylLarginine (L-NMMA) or ADMA in patients with $B C$, as well as their relationship with methylarginine.

\section{Materials and Methods}

\section{Participants and Study Design}

The study included 30 non-muscle invasive bladder cancer (NMIBC) patients and 30 control subjects (group 1) who applied to Manisa Celal Bayar University Faculty of Medicine, Department of Urology, and also patients without malignancy, chronic disease or infection as inclusion criteria. Furthermore, the study involved NMIBC patients with pTa (low grade) and $\geq$ pT2 UCB with transurethral resection of bladder tumour (TURBT) and only intravesical treatment. Blood samples were taken from all patients $(n=30)$ before TURBT (group 2), 20 days after TURBT (group 3) and at the end of intravesical immunotherapy (group 4). All patients and controls who took part in the study provided informed consent, and the study protocol was approved by the Local Ethics Committee.

\section{Methods}

Shimadzu LC-20AD system was used to analyse serum ADMA, SDMA, L-NMMA, arginine and citrulline (16). A total of $200 \mu \mathrm{L}$ of 100 microliters $(\mu \mathrm{L})$ internal standard serum in methanol was added and centrifuged before the supernatant was collected and dried under nitrogen gas. This dried extract was then dissolved in $200 \mu \mathrm{L}$ of a freshly prepared butanol solution containing $5 \%(\mathrm{v} / \mathrm{v})$ acetyl chloride for 20 minutes at $60^{\circ} \mathrm{C}$. This solution was evaporated at $600^{\circ} \mathrm{C}$ using nitrogen gas, then dissolved in $100 \mu \mathrm{L}$ of water-methanol (90:10, v/v) containing $0.1 \%(\mathrm{v} / \mathrm{v})$ formic acid and loaded onto an ultra-performance liquid chromatography analytical column. A total of $40 \mu \mathrm{L}$ was injected. Verieler is built with optimal cone and collision energy values, and the intraday and inter-day coefficients of variation are $8.6 \%$ and $10.1 \%$, respectively.

\section{Statistical Analysis}

When appropriate, data are expressed as the mean \pm standard deviation or means with $95 \%$ confidence interval. The MannWhitney $U$ test was used to examine differences in outcome measures among the groups, and $p<0.05$ was considered statistically significant. Moreover, statistical analysis was done using SPSS software package (15.0; SPSS, Chicago, IL).

\section{Result}

The mean age in the patient group was found to be $67.27 \pm 8.44$, while it was $65.74 \pm 7.22$ in the control group. The difference in mean ages was not statistically significant $(p=0.54)$. In comparison to group 2, ADMA and arginine/total methylated arginine levels in group 1 were significantly lower $(p=0.035$ and $p=0.049$, respectively), while SDMA/ADMA, L-NMMA and arginine/ADMA levels $(p=0.001, p=0.008$ and $p=0.017)$ increased, and no statistical difference was found for other parameters $(p>0.05)$. When compared to group 3, ADMA, arginine, citrulline, methylated arginines and L-NMMA levels in group $2(p=0.035, p=0.001, p=0.015, p=0.032, p=0.032)$ increased, while SDMA/ADMA levels $(p=0.041)$ decreased. Furthermore, ADMA and SDMA levels were higher in group 3, but arginine/ADMA ADMA/total methylarginine were lower than in group 4. Tables 1 and 2 summarise the mean ADMA, SDMA, L-NMMA, arginine, citrulline, arginine/ADMA, SDMA/ ADMA, total methylated arginines and ADMA/methylated arginine levels in each group.

\begin{tabular}{|c|c|c|c|c|c|}
\hline & $\begin{array}{l}\text { ADMA } \\
\text { (uMol/L) }\end{array}$ & $\begin{array}{l}\text { SDMA } \\
\text { (uMol/L) }\end{array}$ & $\begin{array}{l}\text { L-NMMA } \\
\text { (uMol/L) }\end{array}$ & $\begin{array}{l}\text { Arginine } \\
\text { (uMol/L) }\end{array}$ & $\begin{array}{l}\text { Citrulline } \\
\text { (uMol/L) }\end{array}$ \\
\hline Control & 0.196 & 0.211 & 0.26 & 118.57 & 44.55 \\
\hline $\begin{array}{l}\text { Before } \\
\text { TURBT }\end{array}$ & 0.148 & 0.214 & 0.291 & 103.16 & 34.66 \\
\hline $\begin{array}{l}\text { After } \\
\text { TURBT-1 }\end{array}$ & 0.188 & 0.206 & 0.423 & 192.64 & 47.53 \\
\hline $\begin{array}{l}\text { After } \\
\text { TURBT-2 }\end{array}$ & 0.257 & 0.282 & 0.408 & 161 & 50.38 \\
\hline
\end{tabular}

Table 2. Arginine/ADMA ratio, SDMA/ADMA ratio, total methylated arginines, ADMA/total methylated arginine levels in bladder cancer patients

\begin{tabular}{|l|l|l|l|l|}
\hline & $\begin{array}{l}\text { Arginine/ } \\
\text { ADMA } \\
\text { ratio }\end{array}$ & $\begin{array}{l}\text { SDMA/ } \\
\text { ADMA } \\
\text { RATIO }\end{array}$ & $\begin{array}{l}\text { Total } \\
\text { methylated } \\
\text { arginines }\end{array}$ & $\begin{array}{l}\text { ADMA/ } \\
\text { total } \\
\text { methylated } \\
\text { arginines }\end{array}$ \\
\hline Control & 618.47 & 1.04 & 0.67 & 191.5 \\
\hline Before TURBT & 791.5 & 1.57 & 0.65 & 165.78 \\
\hline After TURBT-1 & 1262.39 & 1.2 & 0.82 & 249.81 \\
\hline After TURBT-2 & 800.98 & 1.27 & 0.95 & 185.37 \\
\hline $\begin{array}{l}\text { ADMA: Asymmetrically dimethylated arginine, SDMA: Symmetrically } \\
\text { dimethylated arginine, TURBT: Transurethral resection of bladder tumour }\end{array}$ \\
\hline
\end{tabular}

\section{Discussion}

$\mathrm{BC}$ is one of the most common types of cancers in men. Over $80 \%$ of bladder tumours are (NMIBC, i.e. Tis, Ta or T1), with the remaining $20 \%$ being muscle invasive $B C$ or metastatic BC (17). Most $B C$ are limited to the urothelium and lamina propria, and local treatment has been shown to be effective in many cases. In high-risk patients, an effect is achieved by inducing an immune response through tumour resection and Bacillus Calmette-Guérin to reduce the risk of recurrence (18). Cancer is one of the most common causes of death in the world, with a 
rapidly increasing mechanism due to environmental and genetic factors, the mechanism of which has not been fully disclosed (17).

Today, research is being conducted on signal mechanisms, angiogenesis, apoptosis, metastasis and a variety of other mechanisms in order to gain a better understanding of cancer $(19,20)$. Given the need to improve patient outcomes, it has recently become evident that experimental approaches (including novel chemotherapeutic regimens, biologic agents, immunotherapy and vaccines) are being studied. Arginine modification has been shown to affect DNA repair pathways associated with metastasis and genomic instability (8), while arginine methylation has been shown to be effective in RNA, signal transduction and transcription (21). We aimed to evaluate the levels of arginine, citrulline, ornithine, SDMA, L-NMMA or ADMA in $B C$ patients, as well as their correlation with methylarginine.

Skeletal muscle plasticity has been identified as the regulator (PRMTs), and in vitro studies have shown that these are activated by the methyltransferase pathway, reshaping the muscle remodelling in this way (22). PRMT is an enzyme family that catalyses the addition of one or two methyl groups to the guanidine nitrogen atoms of arginine residues, altering the stability, localisation and/or activity of the labelled molecules (23). Recent research has found that arginine residues in proteins are important for methylation, as well as phosphate groups as control elements in protein functions of methyl groups in mammalian cells $(21,24)$. The protein-DNA complex contains five donors of hydrogen bonds in the arginine structure, which also contains hydrogen bonds with arginine residues.

PRMTs are classified as Type I (PRMT1, 3, 4, 6 and 8) and Type II (PRMT5, 7 and FBXO11) based on their specific catalytic activities (12). S-adenosyl-L-methionine (SAM) is a PRMT family member that is produced by the methionine adenosyltransferase enzyme using methionine and adenosine triphosphate substrates. Moreover, SAM and L-arginine are used by all PRMTs to form the S-adenosylhomocysteine product (22).

As a result of PRMT activity, three types of methylarginines are formed: MMA, SDMA and ADMA (4), where non-specific PRMT produces MMA, type I PRMT generates ADMA, and type II PRMT forms SDMA, all of which are known as PRMT activity indicators $(21,25)$.

PRMT1 and PRMT5 catalyse the reactions that produce ADMA and SDMA, demonstrating enzyme activities (25). The nitric oxide synthase (NOS) family, which includes endothelial NOS (eNOS), neuronal NOS (nNOS) and inducible NOS (iNOS), is responsible for vasodilation and platelet aggregation in response to I-homoarginine (I-hArg). It catalyses the formation of nitric oxide (NO), which is one of its inhibitors, and is inhibited by MMA, ADMA and SDMAs in formed NOs (26). ADMA (13), which is formed by the methylation of arginine in intracellular proteins by type I protein arginine $\mathrm{N}$-methyltransferases, turns into L-citrulline and dimethylarginine when hydrolysed by dimethylaminohydrolase (12). Yoshimatsu et al. (12) discovered that PRMT1, PRMT6 and serum-free ADMA levels increased in various types of cancer. It has been suggested that altering overexpressed PRMT activity in breast, prostate, lung, colon and $\mathrm{BCs}$, as well as leukaemia, may be related to the treatment of these diseases (27). Neault et al. (28) showed that PRMT6 was suppressed indirectly by p21 expression by decreasing p53 gene expression through methylation of PR3T6 in mouse embryonic fibroblasts. Yongchul et al. (8) demonstrated that PRMT6 in the nucleus was stained immunohistochemically positive in colorectal cancers. During the progression of muscle differentiation, Nicole et al. (4) observed an increase in ADMA levels alongside unchanged amounts of MMA and SDMA methylarginine species. The significant up-regulation in ADMA content was consistent with the rise in PRMT1 protein content (4). Despite the fact that Yoshimatsu et al. (12) demonstrated that Type I PRMT expressions increased ADMA serum levels in cancer patients, no studies on BC have been conducted. Any PRMT1 or PRMT6 expression reduction is likely to be beneficial for cancer treatment because it inhibits cancer cell growth (13). We discovered that the mean levels of ADMA and arginine/total methylated arginines in group 2 were significantly lower than those in group 1, while SDMA/ADMA, L-NMMA and arginine/ ADMA levels were higher. Other parameters revealed no statistically significant differences. Moreover, group 2 has higher levels of ADMA, arginine, citrulline, methylated arginines and L-NMMAbut lower levels of SDMA/ADMA than group 3.

\section{Study Limitation}

Our study has some limitations. The first one is that we do not know the long-term outcomes of patients, such as recurrence or progression. Further studies should be conducted to determine the relationship between these parameters and recurrence or progression. The second limitation is that the number of patients was relatively small. The results of our study should be confirmed in future studies involving more patients with BC.

\section{Conclusion}

It is thought that decreases in arginine and ADMA levels in NMIBC patients and these markers can be promising in monitoring patient diagnosis and treatment. Moreover, methylated arginine levels may be useful fin predicting prognosis.

\section{Acknowledgements}

Publication: The results of the study were not published in full or in part in form of abstracts.

Contribution: There is not any contributors who may not be listed as authors.

Conflict of Interest: No conflict of interest was declared by the authors.

Financial Disclosure: This work was supported by Research Fund of Manisa Celal Bayar University.

\section{Ethics}

Ethics Committee Approval: The study protocol was approved by the Local Ethics Committee.

Informed Consent: All patients and controls who took part in the study provided informed consent.

Peer-review: Externally and internally peer-reviewed. 


\section{Authorship Contributions}

Supervision: G.T., Critical Review: G.T., Concept: G.T., F.K., Design: F.K., Data Collection or Processing: G.T., F.K., O.Ü., Analysis or Interpretation: G.T., F.K., S.A., A.Ü., A.S.D., Literature Search: G.T., F.K., Writing: G.T., F.K., Z.A.

\section{References}

1. Luo K-W, Lung W-Y, Xie C, et al. EGCG inhibited bladder cancer T24 and 5637 cell proliferation and migration via PI3K/AKT pathway. Oncotarget 2018;9:12261-12272.

2. Drăgoescu $\mathrm{PO}$, Tudorache Ş, Drocaş $\mathrm{Al}$, et al. Improved diagnosis and long-term recurrence rate reduction for nonmuscle-invasive bladder cancer patients undergoing fluorescent hexylaminolevulinate photodynamic diagnosis. Rom J Morphol Embryol 2017;58:1279-1283.

3. Kim SJ, Yoo BC, Uhm CS, Lee SW. Posttranslational arginine methylation of lamin $\mathrm{A} / \mathrm{C}$ during myoblast fusion. Biochim Biophys Acta 2011;1814:308-317.

4. Nicole YS, Sean YN, Stephen LT, Vladimir L. Protein arginine methyltransferase expression and activity during myogenesis, Biosci Rep 2018;38:BSR20171533. doi: 10.1042/BSR20171533.

5. Stouth DW, van Lieshout TL, Shen NY, Ljubicic V. Regulation of skeletal muscle plasticity by protein arginine methyltransferases and their potential roles in neuromuscular disorders. Front Physiol 2017;8:870.

6. Paik WK, Paik DC, Kim S. Historical review: the field of protein methylation. Trends Biochem Sci 2007;32:146-152.

7. Bedford MT, Richard S. Arginine methylation an emerging regulator of protein function. Mol Cell 2005;18:263-272.

8. Yongchul L, Suyeun $\mathrm{Y}$, Jung-A $\mathrm{Y}$, et al. The prognostic significance of protein arginine methyltransferase 6 expression in colon cancer. Oncotarget 2018;9:9010-9020.

9. McCabe MT, Mohammad HP, Barbash O, Kruger RG. Targeting histone methylation in cancer. Cancer J 2017;23:292-301.

10. Wysocka J, Allis CD, Coonrod S. Histone arginine methylation and its dynamic regulation. Front Biosci 2006;11:344-355.

11. Di Lorenzo A, Bedford MT. Histone arginine methylation. FEBS Lett 2011;585:2024-2031.

12. Yoshimatsu M, Toyokawa G, Hayami S, et al, Dysregulation of PRMT1 and PRMT6, Type I arginine methyltransferases, is involved in various types of human cancers. Int J Cancer 2011;128:562-573.

13. Kielstein JT, Cooke JP. Should we measure asymmetric dimethylarginine in patients with coronary artery disease? Clin Chem 2007;53:161-163.

14. Yang Y, Bedford MT. Protein arginine methyltransferases and cancer. Nat Rev Cancer 2013;13:37-50.
15. Li T, Kong AN, Ma Z, et al. Protein arginine methyltransferase 1 may be involved in pregnane $x$ receptor-activated overexpression of multidrug resistance 1 gene during acquired multidrug resistant. Oncotarget 2016;7:20236-20248. doi: 10.18632/oncotarget.7752.

16. Di Gangi IM, Chiandetti L, Gucciardi A, et al. Simultaneous quantitative determination of $N(G), N(G)$-dimethyl-L-arginine or asymmetric dimethylarginine and related pathway's metabolites in biological fluids by ultrahigh-performance liquid chromatography/ electrospray ionization-tandem mass spectrometry. Anal Chim Acta 2010;677:140-148.

17. Zhang G, Gomes-Giacoia E, Dai Y, et al, Validation and clinicopathologic associations of a urine-based bladder cancer biomarker signature. Diagn Pathol 2014;9:200.

18. Teply BA, Kim JJ. Systemic therapy for bladder cancer - a medical oncologist's perspective, J Solid Tumors 2014;4:25 35.

19. Chen Q, Zhou Z, Shan L, et al. Association of the vascular endothelial growth factor $-2578 \mathrm{c} / \mathrm{a}$ polymorphism with cancer risk: a meta-analysis update. Bıomed Rep 2014;2:823-830.

20. Temeltas $G$, Kosova F, Ucer $O$, et al. Effects of treatment on angiogenic (vascular endothelial growth factor-2 and matrix metalloproteinase-2) and antiangiogenic (endostatin and thrombospondin-1) factors in non-muscle invasive bladder carcinoma. J Uro Surg 2017;4:71-75.

21. Bedford MT, Clarke SG. Protein arginine methylation in mammals: who, what, and why. Mol Cell 2009;33:1-13.

22. Derek W. Stouth, Tiffany L. vanLieshout, Nicole Y. Shen and Vladimir Ljubicic, Regulation of Skeletal Muscle Plasticity by Protein Arginine Methyltransferases and Their Potential Roles in Neuromuscular Disorders, Front Physiol, 2017 Nov 1;8:870.

23. Paik WK, Kim S. Protein methylase I purification and properties of the enzyme. J Biol Chem 1968;243:2108-2114.

24. Najbauer J, Johnson BA, Young AL, Aswad DW. Peptides with sequences similar to glycine, arginine-rich motifs in proteins interacting with RNA are efficiently recognized by methyltransferase(s) modifying arginine in numerous proteins. J Biol Chem 1993;268:10501-10509.

25. Dhar S, Vemulapalli V, Patananan AN, et al. Loss of the major type I arginine methyltransferase PRMT1 causes substrate scavenging by other PRMTs. Sci Rep 2013;3:1311.

26. Tsikas D, Bollenbach A, Hanff E, Kayacelebi AA. Asymmetric dimethylarginine (ADMA), symmetric dimethylarginine (SDMA) and homoarginine (hArg): the ADMA, SDMA and hArg paradoxes. Cardiovasc Diabetol 2018,4;17:1.

27. Baldwin RM, Morettin A, Côté J. Role of PRMTs in cancer: could minor isoforms be leaving a mark? World J Biol Chem 2014;5.115-129.

28. Neault M, Mallette FA, Vogel G, et al. Ablation of PRMT6 reveals a role as a negative transcriptional regulator of the p53 tumor suppressor. Nucleic Acids Res 2012;40:9513-9521. 